\title{
RATIONAL SOLUTIONS OF THE PAINLEVE'-VI EQUATION
}

\author{
M. Mazzocco \\ Mathematical Institute \\ 24-29 St Giles, Oxford, UK.
}

\begin{abstract}
In this paper, we classify all values of the parameters $\alpha, \beta, \gamma$ and $\delta$ of the Painlevé VI equation such that there are rational solutions. We give a formula for them up to the birational canonical transformations and the symmetries of the Painlevé VI equation.
\end{abstract}

\section{Introduction.}

In this paper, we study the general Painlevé sixth equation

$$
\begin{aligned}
y_{x x}= & \frac{1}{2}\left(\frac{1}{y}+\frac{1}{y-1}+\frac{1}{y-x}\right) y_{x}^{2}-\left(\frac{1}{x}+\frac{1}{x-1}+\frac{1}{y-x}\right) y_{x}+ \\
& +\frac{y(y-1)(y-x)}{x^{2}(x-1)^{2}}\left[\alpha+\beta \frac{x}{y^{2}}+\gamma \frac{x-1}{(y-1)^{2}}+\delta \frac{x(x-1)}{(y-x)^{2}}\right],
\end{aligned}
$$

where $x \in \mathbb{C}$ and $\alpha, \beta, \gamma, \delta$ are arbitrary complex parameters. The general solution $y\left(x ; c_{1}, c_{2}\right)$ of the PVI equation satisfies the following two important properties (see [Pain], [Gam]):

1) Painlevé property: the solutions $y\left(x ; c_{1}, c_{2}\right)$ may have complicated singularities (i.e. branch points, essential singularities etc.) only at the critical points $0,1, \infty$ of the equation (the so-called fixed singularities). All the other singularities, the position of which depend on the integration constants (the so-called movable singularities), are poles.

2) For generic values of the integration constants $c_{1}, c_{2}$ and of the parameters $\alpha, \beta, \gamma, \delta$, the solution $y\left(x ; c_{1}, c_{2}\right)$ can not be expressed via known functions.

The latter property needs to be stated more precisely. Following [Um1], [Um2], we define known or classical functions to be functions that can be obtained from the field of rational functions $\mathbb{C}(x)$, by a finite iteration of the following operations:

i) derivation,

ii) quadrature,

iii) arithmetic operations,,$+- \times, \div$,

iv) solution of a homogeneous linear ordinary differential equations with classical coefficients,

v) substitution into an Abelian function,

vi) solution of algebraic differential equations of first order with classical coefficients.

According to this definition, Watanabe (see [Wat]) proved that for generic values of the integration constants $c_{1}, c_{2}$ and of the parameters $\alpha, \beta, \gamma, \delta$, the solutions $y\left(x ; c_{1}, c_{2}\right)$ of PVI are non-classical and classified all the one-parameter families of classical solutions of PVI. Loosely speaking, Watanabe proves that all oneparameter families of classical solutions of PVI are realized for values of the parameters $\alpha, \beta, \gamma, \delta$ lying on the walls of the Weyl chamber of the group $\tilde{W}$ of the birational canonical transformations. ${ }^{1}$ Such one-parameter families of classical solutions, already found by Okamoto, shrink down, by the action of the group $\tilde{W}$, to the following list ${ }^{2}$

i) $y(x) \equiv \infty$, for $\alpha=0$,

ii) $y(x) \equiv 0$, for $\beta=0$,

iii) $y(x) \equiv 1$, for $\gamma=0$,

iv) $y(x) \equiv x$, for $\delta=\frac{1}{2}$,

1 Recall that $\tilde{W}$ is isomorphic to $W_{a}\left(D_{4}\right)$ the affine extension of the Weyl group of $D_{4}$, (see [Ok]).

2 The group $\tilde{W}$ acts on $y(x)$ and on its conjugate momentum $p(x)$. In the list i)..,iv) the conjugate momentum $p(x)$ is given by a one-parameter family. 
v) Riccati solutions,

$$
\frac{\mathrm{d}}{\mathrm{d} x} y=-\frac{y(y-1)(y-x)}{x(x-1)}\left(\frac{\vartheta_{1}}{y}+\frac{\vartheta_{2}-1}{y-x}+\frac{\vartheta_{3}}{y-1}\right),
$$

for $\vartheta_{\infty}=\vartheta_{1}+\vartheta_{2}+\vartheta_{3}$, where $\vartheta_{\infty}, \vartheta_{1}, \vartheta_{2}, \vartheta_{3}$ are defined by

$$
\alpha=\frac{\left(\vartheta_{\infty}-1\right)^{2}}{2}, \quad \beta=-\frac{\vartheta_{1}^{2}}{2}, \quad \gamma=\frac{\vartheta_{3}^{2}}{2}, \quad \delta=\frac{1-\vartheta_{2}^{2}}{2} .
$$

Solutions (i), (ii), (iii), (iv) are called degenerate.

The theory of the rational and classical solutions of the Painlevé sixth equation has been extensively studied in $[\mathrm{Ai}],[\mathrm{AMM}],[\mathrm{Gr}],[\mathrm{GL}],[\mathrm{Luk}],[\mathrm{Um}]$, [Wat]. In this paper we classify all rational solutions of PVI. We prove the following

Main Theorem. All rational non-degenerate solutions of PVI belong to the intersection of two or more one-parameter families of classical solutions, i.e. they occur for

$$
\vartheta_{\infty}+\sum_{k=1}^{3} \varepsilon_{k} \vartheta_{k} \in 2 \mathbb{Z}, \quad \varepsilon_{k}= \pm 1, \quad \text { and } \quad \vartheta_{k} \in \mathbb{Z} \text { for at least one } k=1,2,3, \infty .
$$

All rational non-degenerate solutions of the PVI equation are equivalent via birational canonical transformations and up to symmetries ${ }^{2}$ to the following solutions

$$
\begin{gathered}
y(x)=\frac{(x}{\left(1+\vartheta_{2}+x+x \vartheta_{3}\right)}, \quad \text { for } \quad \vartheta_{\infty}=-\sum_{k=1}^{3} \vartheta_{k} \quad \text { and } \quad \vartheta_{1}=1, \\
y(x)=\frac{\left(\vartheta_{2}+\vartheta_{3} x\right)^{2}-\vartheta_{2}-\vartheta_{3} x^{2}}{\left(\vartheta_{2}+\vartheta_{3}-1\right)\left(\vartheta_{2}+\vartheta_{3} x\right)}, \quad \text { for } \quad \vartheta_{\infty}=-\sum_{k=1}^{3} \vartheta_{k} \quad \text { and } \quad \vartheta_{1}=-2 .
\end{gathered}
$$

Our method to prove this result does not use Umemura's theory, but the isomonodromy deformation method (see [Fuchs], [Sch], [JMU], [ItN], [FlN]). The Painlevé VI is represented as the equation of isomonodromy deformation of the two-dimensional auxiliary Fuchsian system

$$
\frac{\mathrm{d}}{\mathrm{d} \lambda} \Phi=\left(\frac{\mathcal{A}_{1}}{\lambda-u_{1}}+\frac{\mathcal{A}_{2}}{\lambda-u_{2}}+\frac{\mathcal{A}_{3}}{\lambda-u_{3}}\right) \Phi
$$

$\mathcal{A}_{j}$ being $2 \times 2$ matrices independent on $\lambda$, and $u_{1}, u_{2}, u_{3}$ being pairwise distinct complex numbers. The matrices $\mathcal{A}_{j}$ satisfy the following conditions:

$$
\text { eigen }\left(\mathcal{A}_{j}\right)= \pm \frac{\vartheta_{j}}{2} \quad \text { and } \quad-\mathcal{A}_{1}-\mathcal{A}_{2}-\mathcal{A}_{3}=\mathcal{A}_{\infty}:=\frac{1}{2}\left(\begin{array}{cc}
\vartheta_{\infty} & 0 \\
0 & -\vartheta_{\infty}
\end{array}\right)
$$

$\vartheta_{j}, j=1,2,3, \infty$ being related to the parameters $\alpha, \beta, \gamma, \delta$ of PVI as in (1.2).

The entries of the matrices $A_{i}$ are complicated expressions of $x, y, y_{x}$ and of some quadrature $\int R(x, y) \mathrm{d} x$, $x$ being the cross ratio of the poles $x=\frac{u_{2}-u_{1}}{u_{3}-u_{1}}$. The monodromy matrices $\mathcal{M}_{1}, \mathcal{M}_{2}$ and $\mathcal{M}_{3}$ of (1.3) remain constant if and only if $y=y(x)$ satisfies PVI. To each branch of a solution of PVI corresponds a triple $\mathcal{M}_{1}, \mathcal{M}_{2}, \mathcal{M}_{3}$ of monodromy matrices, which is unique up to

$$
\left(\mathcal{M}_{1}, \mathcal{M}_{2}, \mathcal{M}_{3}\right) \rightarrow L_{\infty}^{-1}\left(\mathcal{M}_{1}, \mathcal{M}_{2}, \mathcal{M}_{3}\right) L_{\infty}
$$

where $L_{\infty}$ is any constant invertible matrix such that $\left[L_{\infty}, \mathcal{M}_{\infty}\right]=0$ (see Section 2).

2 The symmetries of the Painlevé VI equation are compositions of the following transformations i) $x \rightarrow$ $1-x, y \rightarrow 1-y, \vartheta_{1} \leftrightarrow \vartheta_{3}$, ii) $x \rightarrow \frac{1}{x}, y \rightarrow \frac{1}{y}, \vartheta_{\infty} \leftrightarrow \vartheta_{1}+1$, iii) $x \rightarrow \frac{1}{1-x}, y \rightarrow \frac{q-x}{x-1}, \vartheta_{1} \leftrightarrow \vartheta_{2}$. 
Following the same strategy as in $[\mathrm{DM}]$, we describe the procedure of analytic continuation of a branch of a solution of PVI by the action of the pure braid group on its monodromy matrices. Since rational solutions have only one branch, we look for fixed points of this action. We show that a necessary condition to have a rational (non-degenerate) solution is that the corresponding monodromy group is abelian (see Section 3).

Abelian $2 \times 2$ monodromy groups are reducible. In Section 4, we classify all solutions of PVI having a reducible monodromy group (such solutions where found in [Hit] as a reduction of Nahm's equations for a diffeomorphic group). Then we classify all rational solutions among them.

Acknowledgements. The idea of classifying all rational solutions of PVI came out of a conversation with H. Umemura. I am indebted to B. Dubrovin who introduced me to the theory of Painlevé equations and gave me lots of suggestions. I am grateful to N. Hitchin, who constantly addressed my work and A.C.C. Coolen for kindly hosting me at Kings College London where this paper was started. The author was supported by an EPSRC research assistanship. We thank P. Clarkson and P. Boalch for pointing out some of the typos which have been fixed in this version.

\section{The Painlevé VI equation as the isomonodromic deformation equation of a $2 \times 2$ Fuchsian system.}

Consider the following Fuchsian system with four pairwise distinct regular singularities at $u_{1}, u_{2}, u_{3}, \infty$ :

$$
\frac{\mathrm{d}}{\mathrm{d} \lambda} \Phi=\left(\frac{\mathcal{A}_{1}}{\lambda-u_{1}}+\frac{\mathcal{A}_{2}}{\lambda-u_{2}}+\frac{\mathcal{A}_{3}}{\lambda-u_{3}}\right) \Phi, \quad \lambda \in \overline{\mathbb{C}} \backslash\left\{u_{1}, u_{2}, u_{3}, \infty\right\}
$$

$\mathcal{A}_{j}$ being $2 \times 2$ matrices independent of $\lambda$. Assume that the matrices $\mathcal{A}_{j}$ satisfy the following conditions:

$$
\text { eigen }\left(\mathcal{A}_{j}\right)= \pm \frac{\vartheta_{j}}{2} \quad \text { and } \quad-\mathcal{A}_{1}-\mathcal{A}_{2}-\mathcal{A}_{3}=\mathcal{A}_{\infty}
$$

for some constants $\vartheta_{j}, j=1,2,3$ and

$$
\mathcal{A}_{\infty}:=\frac{1}{2}\left(\begin{array}{cc}
\vartheta_{\infty} & 0 \\
0 & -\vartheta_{\infty}
\end{array}\right), \quad \text { for some constant } \vartheta_{\infty} \neq 0
$$

The solution $\Phi(\lambda)$ of the system (2.1) is a multi-valued analytic function on $\mathbb{C} \backslash\left\{u_{1}, u_{2}, u_{3}\right\}$, and its multivaluedness is described by the monodromy matrices. To define them, we fix a basis $\gamma_{1}, \gamma_{2}, \gamma_{3}$ of loops in $\pi_{1}\left(\overline{\mathbb{C}} \backslash\left\{u_{1}, u_{2}, u_{3}, \infty\right\}, \infty\right)$ as in figure 1 , and a fundamental matrix for the system (2.1).

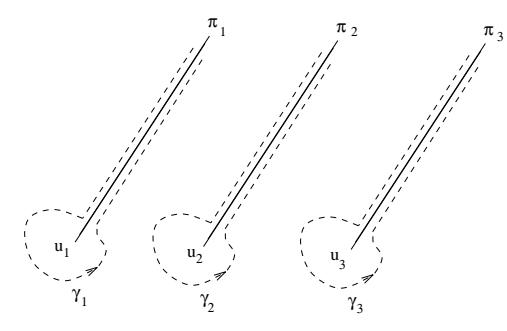

Fig. 1. The branch-cuts $\pi_{j}$ between the ordered singularities $u_{j}$ and the oriented loops $\gamma_{j}$ in the $\lambda$-plane. 
Proposition 2.1. There exists a fundamental matrix of the system (2.1) of the form

$$
\Phi_{\infty}(\lambda)=\left(\mathbf{1}+\mathcal{O}\left(\frac{1}{\lambda}\right)\right) \lambda^{-\mathcal{A}_{\infty}} \lambda^{-\mathcal{R}_{\infty}}, \quad \text { as } \quad \lambda \rightarrow \infty,
$$

where $\lambda^{-\mathcal{R}_{\infty}}:=e^{-\mathcal{R}_{\infty} \log \lambda}$, with the choice of the principal branch of the logarithm with the branch-cut along the common direction $\eta$ of the cuts $\pi_{1}, \pi_{2}, \pi_{3}$ and the matrix entries of $\mathcal{R}_{\infty}$ given by $\mathcal{R}_{\infty 11}=\mathcal{R}_{\infty 22}=0$ and

$$
\begin{aligned}
& \text { for } \vartheta_{\infty} \notin \mathbb{Z}, \text { and for } \vartheta_{\infty}=0, \quad \mathcal{R}_{\infty 12}=\mathcal{R}_{\infty 21}=0, \\
& \text { for } \vartheta_{\infty}=n \in \mathbb{Z}_{+}, \quad \mathcal{R}_{\infty 12}=\mathcal{R}_{\infty 12}\left(\mathcal{A}_{1,2,3}, u_{1,2,3}\right), \quad \mathcal{R}_{\infty 21}=0, \\
& \text { for } \vartheta_{\infty}=-n, n \in \mathbb{Z}_{+}, \quad \mathcal{R}_{\infty 21}=\mathcal{R}_{\infty 21}\left(\mathcal{A}_{1,2,3}, u_{1,2,3}\right), \quad \mathcal{R}_{\infty 12}=0,
\end{aligned}
$$

where the functions $\mathcal{R}_{\infty 12,21}\left(\mathcal{A}_{1,2,3}, u_{1,2,3}\right)$ are uniquely determined by $\left(\mathcal{A}_{1,2,3}, u_{1,2,3}\right)$. Such a fundamental matrix $\Phi_{\infty}(\lambda)$ is uniquely determined up to

$$
\Phi_{\infty}(\lambda) \rightarrow \Phi_{\infty}(\lambda) L_{\infty}
$$

where $L_{\infty}$ is any constant invertible matrix such that

$$
\lambda^{-\mathcal{A}_{\infty}} \lambda^{-\mathcal{R}_{\infty}} L_{\infty} \lambda^{\mathcal{A}_{\infty}} \lambda^{\mathcal{R}_{\infty}}=\mathbf{1}+\sum_{k=1}^{N} \frac{L_{\infty}^{(k)}}{\lambda^{k}}
$$

for some $L_{\infty}^{(1)}, \cdots, L_{\infty}^{(N)}$ constant matrices.

The proof can be found in [Dub].

The fundamental matrix $\Phi_{\infty}$ can be analytically continued to an analytic function on the universal covering of $\overline{\mathbb{C}} \backslash\left\{u_{1}, u_{2}, u_{3}, \infty\right\}$. For any element $\gamma \in \pi_{1}\left(\overline{\mathbb{C}} \backslash\left\{u_{1}, u_{2}, u_{3}, \infty\right\}, \infty\right)$ denote the result of the analytic continuation of $\Phi_{\infty}(\lambda)$ along the loop $\gamma$ by $\gamma\left[\Phi_{\infty}(\lambda)\right]$. Since $\gamma\left[\Phi_{\infty}(\lambda)\right]$ and $\Phi_{\infty}(\lambda)$ are two fundamental matrices in the neighbourhood of infinity, they are related by the following relation:

$$
\gamma\left[\Phi_{\infty}(\lambda)\right]=\Phi_{\infty}(\lambda) \mathcal{M}_{\gamma}
$$

for some constant invertible $2 \times 2$ matrix $\mathcal{M}_{\gamma}$ depending only on the homotopy class of $\gamma$. Particularly, the matrix $\mathcal{M}_{\infty}:=\mathcal{M}_{\gamma_{\infty}}, \gamma_{\infty}$ being a simple loop around infinity in the clock-wise direction, is given by:

$$
\mathcal{M}_{\infty}=\exp \left(2 \pi i \mathcal{A}_{\infty}\right) \exp \left(2 \pi i \mathcal{R}_{\infty}\right) .
$$

The resulting monodromy representation is an anti-homomorphism:

$$
\begin{aligned}
\pi_{1}\left(\overline{\mathbb{C}} \backslash\left\{u_{1}, u_{2}, u_{3}, \infty\right\}, \infty\right) & \rightarrow S L_{2}(\mathbb{C}) \\
\gamma & \mapsto \mathcal{M}_{\gamma} \\
\mathcal{M}_{\gamma \tilde{\gamma}}=\mathcal{M}_{\tilde{\gamma}} \mathcal{M}_{\gamma} . &
\end{aligned}
$$

The images $\mathcal{M}_{j}:=\mathcal{M}_{\gamma_{j}}$ of the generators $\gamma_{j}, j=1,2,3$ of the fundamental group, are called the monodromy matrices of the Fuchsian system (2.1). They generate the monodromy group of the system, i.e. the image of the representation (2.9). Since the loop $\left(\gamma_{1} \gamma_{2} \gamma_{3}\right)^{-1}$ is homotopic to $\gamma_{\infty}$, the following relation between the generators holds:

$$
\mathcal{M}_{\infty} \mathcal{M}_{3} \mathcal{M}_{2} \mathcal{M}_{1}=1
$$

Observe that if we fix another fundamental matrix $\Phi_{\infty}^{\prime}=\Phi_{\infty} L_{\infty}$ in the equivalence class defined by (2.6), the monodromy matrices $\mathcal{M}_{\gamma}^{\prime}$ with respect to the new fundamental matrix $\Phi_{\infty}^{\prime}$ are related to the old ones by

$$
\mathcal{M}_{j}^{\prime}=L_{\infty}^{-1} \mathcal{M}_{j} L_{\infty}, \quad j=1,2,3 .
$$

Thus the monodromy matrices $\mathcal{M}_{3}, \mathcal{M}_{2}, \mathcal{M}_{1}$ are uniquely defined up to the ambiguity

$$
\left(\mathcal{M}_{1}, \mathcal{M}_{2}, \mathcal{M}_{3}\right) \sim\left(L_{\infty}^{-1} \mathcal{M}_{1} L_{\infty}, L_{\infty}^{-1} \mathcal{M}_{2} L_{\infty}, L_{\infty}^{-1} \mathcal{M}_{3} L_{\infty}\right)
$$

where $L_{\infty}$ is given by (2.7). Observe that $\mathcal{M}_{\infty}$ is invariant w.r.t. (2.12).

I recall the definition of the connection matrices. Near the poles $u_{j}$, the fundamental matrices $\Phi_{j}(\lambda)$ of the system (2.1), are given by the following 
Proposition 2.2. There exists a fundamental matrix of the system (2.1) of the form

$$
\Phi_{j}(\lambda)=G_{j}\left(\mathbf{1}+\mathcal{O}\left(\lambda-u_{j}\right)\right)\left(\lambda-u_{j}\right)^{J_{j}}\left(\lambda-u_{j}\right)^{\mathcal{R}_{j}}, \quad \text { as } \quad \lambda \rightarrow u_{j}
$$

where

$$
\begin{array}{ll}
\text { for } \vartheta_{j} \neq 0, & J_{j}=\frac{1}{2}\left(\begin{array}{cc}
\vartheta_{j} & 0 \\
0 & -\vartheta_{j}
\end{array}\right), \\
\text { for } \vartheta_{j}=0, & J_{j}=J \equiv\left(\begin{array}{ll}
0 & 1 \\
0 & 0
\end{array}\right) .
\end{array}
$$

The invertible matrix $G_{j}$ is defined by $\mathcal{A}_{j}=G_{j} J_{j} G_{j}^{-1}$, the diagonal elements of the matrix $\mathcal{R}_{j}$ are zero and the off-diagonal ones are defined as follows,

$$
\begin{aligned}
& \text { for } \vartheta_{j} \notin \mathbb{Z}, \text { and for } \vartheta_{j}=0 \quad \mathcal{R}_{j_{12}}=\mathcal{R}_{j_{21}}=0, \\
& \text { for } \vartheta_{j}=n \in \mathbb{Z}_{+}, \quad \mathcal{R}_{j_{12}}=\mathcal{R}_{j_{12}}\left(\mathcal{A}_{1,2,3}, u_{1,2,3}\right), \quad \mathcal{R}_{j_{21}}=0, \\
& \text { for } \vartheta_{j}=-n, n \in \mathbb{Z}_{+}, \quad \mathcal{R}_{j_{21}}=\mathcal{R}_{j_{21}}\left(\mathcal{A}_{1,2,3}, u_{1,2,3}\right), \quad \mathcal{R}_{j_{12}}=0
\end{aligned}
$$

where the functions $\mathcal{R}_{j_{21,12}}\left(\mathcal{A}_{1,2,3}, u_{1,2,3}\right)$ are uniquely determined by $\mathcal{A}_{1,2,3}$ and $u_{1,2,3}$. The choice of the branch of $\log \left(z-u_{j}\right)$ is along $\eta$ as above. The fundamental matrix $\Phi_{j}(\lambda)$ is uniquely determined up to the ambiguity:

$$
\Phi_{j}(\lambda) \mapsto \Phi_{j}(\lambda) L_{j}
$$

where $L_{j}$ is any constant invertible matrix such that

$$
\left(\lambda-u_{j}\right)^{J_{j}}\left(\lambda-u_{j}\right)^{\mathcal{R}_{j}} L_{j}\left(\lambda-u_{j}\right)^{-J_{j}}\left(\lambda-u_{j}\right)^{-\mathcal{R}_{j}}=\sum_{k=0}^{N} L_{j}^{(k)}\left(\lambda-u_{j}\right)^{k}
$$

for $L_{j}^{(0)}=G_{j}$ and for some $L_{j}^{(1)}, \cdots, L_{j}^{(N)}$ constant matrices.

The proof can be found in [Dub].

Continuing the solution $\Phi_{\infty}(\lambda)$ to a neighbourhood of $u_{j}$, along, say, the right-hand-side of the branchcut $\pi_{j}$, one obtains another fundamental matrix around $u_{j}$, that must be related to $\Phi_{j}(\lambda)$ by:

$$
\Phi_{\infty}(\lambda)=\Phi_{j}(\lambda) \mathcal{C}_{j}
$$

for some invertible matrix $\mathcal{C}_{j}$. The matrices $\mathcal{C}_{1}, \mathcal{C}_{2}, \mathcal{C}_{3}$ are called connection matrices, and they are defined by (2.17) up to the ambiguity $\mathcal{C}_{j} \rightarrow \mathcal{C}_{j} L_{\infty}$ due to (2.6). The connection matrices are related to the monodromy matrices as follows:

$$
\mathcal{M}_{j}=\mathcal{C}_{j}^{-1} \exp \left(2 \pi i J_{j}\right) \exp \left(\mathcal{R}_{j}\right) \mathcal{C}_{j}, \quad j=1,2,3 .
$$

Thanks to the above relation it follows that

$$
\operatorname{eigen}\left(\mathcal{M}_{j}\right)=\exp \left( \pm \pi i \vartheta_{j}\right)
$$

Definition 2.3. The Monodromy data of the Fuchsian system (2.1) are

$$
\left\{\left(\mathcal{M}_{1}, \mathcal{M}_{2}, \mathcal{M}_{3}\right) / \sim, \mathcal{R}_{1}, \mathcal{R}_{2}, \mathcal{R}_{3}\right\}
$$

where $\sim$ is the equivalence relation defined by (2.12).

Remark 2.4. For non-integer $\vartheta_{j}$, the correspondent $\mathcal{R}_{j}$ matrix is zero by definition and we drop it from the set of the monodromy data.

The theory of the deformations the poles of the Fuchsian system keeping the monodromy fixed is described by the following result: 
Theorem 2.5. Let $\left\{\left(\mathcal{M}_{1}, \mathcal{M}_{2}, \mathcal{M}_{3}\right) / \sim, \mathcal{R}_{1}, \mathcal{R}_{2}, \mathcal{R}_{3}\right\}$, be monodromy data of the Fuchsian system:

$$
\frac{\mathrm{d}}{\mathrm{d} \lambda} \Phi^{0}=\left(\frac{\mathcal{A}_{1}^{0}}{\lambda-u_{1}^{0}}+\frac{\mathcal{A}_{2}^{0}}{\lambda-u_{2}^{0}}+\frac{\mathcal{A}_{3}^{0}}{\lambda-u_{3}^{0}}\right) \Phi^{0}
$$

of the above form (2.2), with pairwise distinct poles $u_{j}^{0}$, and with respect to some basis $\gamma_{1}, \gamma_{2}, \gamma_{3}$ of the loops in $\pi_{1}\left(\overline{\mathbb{C}} \backslash\left\{u_{1}^{0}, u_{2}^{0}, u_{3}^{0}, \infty\right\}, \infty\right)$. If $\mathcal{M}_{k} \neq \pm \mathbf{1}$ for all $k=1,2,3, \infty$, there exists a neighbourhood $U \subset \mathbb{C}^{3}$ of the point $u^{0}=\left(u_{1}^{0}, u_{2}^{0}, u_{3}^{0}\right)$ such that, for any $u=\left(u_{1}, u_{2}, u_{3}\right) \in U$, there exists a unique triple $\mathcal{A}_{1}(u), \mathcal{A}_{2}(u)$, $\mathcal{A}_{3}(u)$ of analytic matrix valued functions such that:

$$
\mathcal{A}_{j}\left(u^{0}\right)=\mathcal{A}_{j}^{0}, \quad i=1,2,3,
$$

and the monodromy matrices of the Fuchsian system

$$
\frac{\mathrm{d}}{\mathrm{d} \lambda} \Phi=A(\lambda ; u) \Phi=\left(\frac{\mathcal{A}_{1}(u)}{\lambda-u_{1}}+\frac{\mathcal{A}_{2}(u)}{\lambda-u_{2}}+\frac{\mathcal{A}_{3}(u)}{\lambda-u_{3}}\right) \Phi,
$$

with respect to the same basis ${ }^{1} \gamma_{1}, \gamma_{2}, \gamma_{3}$ of the loops, can be chosen to coincide with the given $\mathcal{M}_{1}, \mathcal{M}_{2}$, $\mathcal{M}_{3}$. The matrices $\mathcal{A}_{j}(u)$ are the solutions of the Cauchy problem with the initial data $\mathcal{A}_{j}^{0}$ for the following Schlesinger equations:

$$
\frac{\partial}{\partial u_{j}} \mathcal{A}_{i}=\frac{\left[\mathcal{A}_{i}, \mathcal{A}_{j}\right]}{u_{i}-u_{j}}, \quad \frac{\partial}{\partial u_{i}} \mathcal{A}_{i}=-\sum_{j \neq i} \frac{\left[\mathcal{A}_{i}, \mathcal{A}_{j}\right]}{u_{i}-u_{j}} .
$$

The solution $\Phi_{\infty}^{0}(\lambda)$ of (2.20) of the form (2.4) can be uniquely continued, for $\lambda \neq u_{i} i=1,2,3$, to an analytic function $\Phi_{\infty}(\lambda, u), \quad u \in U$, such that

$$
\Phi_{\infty}\left(\lambda, u^{0}\right)=\Phi_{\infty}^{0}(\lambda)
$$

This continuation is the local solution of the Cauchy problem with the initial data $\Phi_{\infty}^{0}$ for the following system that is compatible with the system (2.21):

$$
\frac{\partial}{\partial u_{i}} \Phi=-\frac{\mathcal{A}_{i}(u)}{\lambda-u_{i}} \Phi
$$

Moreover the functions $\mathcal{A}_{i}(u)$ and $\Phi_{\infty}(\lambda, u)$ can be continued analytically to global meromorphic functions on the universal coverings of

$$
\mathbb{C}^{3} \backslash\{\text { diags }\}:=\left\{\left(u_{1}, u_{2}, u_{3}\right) \in \mathbb{C}^{3} \mid u_{i} \neq u_{j} \text { for } i \neq j\right\},
$$

and

$$
\left\{\left(\lambda, u_{1}, u_{2}, u_{3}\right) \in \mathbb{C}^{4} \mid u_{i} \neq u_{j} \text { for } i \neq j \text { and } \lambda \neq u_{i}, i=1,2,3\right\},
$$

respectively.

The proof of this theorem can be found, for example, in [Mal], [Miwa], [Sib].

Remark 2.6. Observe that the isomonodromic deformations equations preserve the connection matrices $\mathcal{C}_{i}$ too.

Remark 2.7. When $\mathcal{M}_{k}= \pm \mathbf{1}$ for some $k=1,2,3 \infty$, the existence statements of Theorem 2.5 are still valid, while the uniqueness ones are lost.

1 Observe that the basis $\gamma_{1}, \gamma_{2}, \gamma_{3}$ of $\pi_{1}\left(\overline{\mathbb{C}} \backslash\left\{u_{1}, u_{2}, u_{3}, \infty\right\}, \infty\right)$ varies continuously with small variations of $u_{1}, u_{2}, u_{3}$. This new basis is homotopic to the initial one, so one can identify them. 
Let me now explain, following [JMU], how to rewrite the Schlesinger equations (2.22) in terms of the PVI equation. We can assume $\vartheta_{\infty} \neq 0$ without loss of generality (see Remark 2.9 below). Schlesinger equations (2.22) with fixed $\mathcal{A}_{\infty}$ are invariant with respect to the gauge transformations of the form:

$$
\mathcal{A}_{i} \mapsto D^{-1} \mathcal{A}_{i} D, \quad i=1,2,3, \quad \text { for any } D \text { diagonal matrix. }
$$

We introduce two coordinates $(p, q)$ on the quotient of the space of matrices satisfying (2.22) with respect to the equivalence relation $(2.23)$ and a coordinate $k$ that contains the above gauge freedom:

$$
\left[\mathcal{A}\left(q ; u_{1}, u_{2}, u_{3}\right)\right]_{12}=0, \quad p=\sum_{k=1}^{3} \frac{\mathcal{A}_{k_{11}}+\frac{\vartheta_{k}}{2}}{q-u_{k}}, \quad k=\frac{2 P(\lambda)\left[\mathcal{A}\left(\lambda ; u_{1}, u_{2}, u_{3}\right)\right]_{12}}{\vartheta_{\infty}(q-\lambda)},
$$

where $\mathcal{A}\left(z ; u_{1}, u_{2}, u_{3}\right)$ is given in $(2.21)$ and $P(\lambda)=\left(\lambda-u_{1}\right)\left(\lambda-u_{2}\right)\left(\lambda-u_{3}\right)$. The matrices $\mathcal{A}_{i}$ are uniquely determined by the coordinates $(p, q)$, and $k$ and expressed rationally in terms of them:

$$
\begin{aligned}
\mathcal{A}_{i 11} & =\frac{1}{\vartheta_{\infty} P^{\prime}\left(u_{i}\right)}\left\{P(q)\left(q-u_{i}\right) p^{2}+P(q)\left(q-u_{i}\right) p\left(\frac{\vartheta_{\infty}}{q-u_{i}}-\sum_{k=1}^{3} \frac{\vartheta_{k}}{q-u_{k}}\right)+\right. \\
& +\left(q-u_{i}\right)\left[\frac{\vartheta_{\infty}^{2}}{4}\left(q+2 u_{i}-\sum_{k=1}^{3} u_{k}\right)+\sum_{k=1}^{3} \frac{\vartheta_{k}^{2}}{4}\left(q+2 u_{k}-\sum_{j=1}^{3} u_{j}\right)\right]+ \\
& \left.+\frac{q-u_{i}}{2}\left(\vartheta_{1} \vartheta_{2}\left(q-u_{3}\right)+\vartheta_{1} \vartheta_{3}\left(q-u_{2}\right)+\vartheta_{2} \vartheta_{3}\left(q-u_{1}\right)\right)-\frac{\vartheta_{\infty}}{2} \sum_{k=1}^{3} \frac{\vartheta_{k}}{q-u_{k}}\right\} \\
\mathcal{A}_{i 12} & =-\vartheta_{\infty} k \frac{q-u_{i}}{2 P^{\prime}\left(u_{i}\right)} \\
\mathcal{A}_{i 21} & =\frac{1}{\mathcal{A}_{i 12}}\left(\frac{\vartheta_{i}^{2}}{4}-\mathcal{A}_{i 11}^{2}\right) \\
\mathcal{A}_{i 22} & =-\mathcal{A}_{i 11}
\end{aligned}
$$

for $i=1,2,3$, where $P^{\prime}(z)=\frac{\mathrm{d} P}{\mathrm{~d} z}$. The Schlesinger equations (2.22) in these variables are:

$$
\left\{\begin{aligned}
\frac{\partial q}{\partial u_{i}} & =\frac{P(q)}{P^{\prime}\left(u_{i}\right)}\left[2 p+\frac{1}{q-u_{i}}-\sum_{k=1}^{3} \frac{\vartheta_{k}}{q-u_{k}}\right] \\
\frac{\partial p}{\partial u_{i}} & =-\left\{P^{\prime}(q) p^{2}+\left[2 q+u_{i}-\sum_{j} u_{j}-\sum_{k=1}^{3} \vartheta_{k}\left(2 q+u_{k}-\sum_{j} u_{j}\right)\right] p+\right. \\
& \left.+\frac{1}{4}\left(\sum_{k=1}^{3} \vartheta_{k}-\vartheta_{\infty}\right)\left(\sum_{k=1}^{3} \vartheta_{k}+\vartheta_{\infty}-2\right)\right\} \frac{1}{P^{\prime}\left(u_{i}\right)}
\end{aligned}\right.
$$

and

$$
\frac{\partial \log (k)}{\partial u_{i}}=\left(\vartheta_{\infty}-1\right) \frac{q-u_{i}}{P^{\prime}\left(u_{i}\right)} .
$$

for $i=1,2,3$. The system of the reduced Schlesinger equations (2.25) is invariant under the transformations of the form

$$
u_{i} \mapsto a u_{i}+b, \quad q \mapsto a q+b, \quad p \mapsto \frac{p}{a}, \quad \forall a, b \in \mathbb{C}, \quad a \neq 0 .
$$

Introducing the following new invariant variables:

$$
x=\frac{u_{2}-u_{1}}{u_{3}-u_{1}}, \quad y=\frac{q-u_{1}}{u_{3}-u_{1}}
$$


the system (2.25), expressed in the these new variables, gives the PVI equation for $y(x)$ with parameters

$$
\alpha=\frac{\left(\vartheta_{\infty}-1\right)^{2}}{2}, \quad \beta=-\frac{\vartheta_{1}^{2}}{2}, \quad \gamma=\frac{\vartheta_{3}^{2}}{2}, \quad \delta=\frac{1-\vartheta_{2}^{2}}{2} .
$$

Remark 2.8. Observe that permutations of the poles $u_{i}$ and of the values $\vartheta_{i}, i=1,2,3, \infty$ induce transformations of $(y, x)$ of the type $x \rightarrow 1-x$ and $y \rightarrow 1-y, x \rightarrow \frac{1}{x}$ and $y \rightarrow \frac{1}{y}, x \rightarrow \frac{1}{1-x}$ and $y \rightarrow \frac{y-x}{1-x}$ and their compositions. These transformations are the symmetries of the Painlevè VI equation.

Remark 2.9. It is clear from (2.28) that changes of the signs of the parameters $\vartheta_{k}, k=1,2,3$ and transformations on $\vartheta_{\infty}$ of type $\vartheta_{\infty} \rightarrow 2-\vartheta_{\infty}$ give rise to the same PVI equation.

We summarise the results of this section in the following:

Theorem 2.10. Branches $y(x)$ of solutions of the PVI equation with parameters $\alpha, \beta, \gamma, \delta$, considered up to symmetries are in one to one correspondence with local solutions of the Schlesinger equations (2.22) with parameters $\vartheta_{1}, \vartheta_{2}, \vartheta_{3}, \vartheta_{\infty}$ given by (2.28) and $\mathcal{A}_{\infty}$ given in (2.3), considered up to diagonal conjugation (2.23) and permutation. This one-to-one correspondence is realized by the formulae (2.24), (2.27). For each branch of a solution of the PVI equation, there exist a unique set of monodromy data $\left\{\left(\mathcal{M}_{1}, \mathcal{M}_{2}, \mathcal{M}_{3}\right) / \sim, \mathcal{R}_{1}, \mathcal{R}_{2}, \mathcal{R}_{3}\right\}$. Vice-versa, let $\left\{\left(\mathcal{M}_{1}, \mathcal{M}_{2}, \mathcal{M}_{3}\right) / \sim, \mathcal{R}_{1}, \mathcal{R}_{2}, \mathcal{R}_{3}\right\}$ be a set of monodromy data such that

$$
\begin{gathered}
\text { eigen }\left(\mathcal{M}_{j}\right)=\exp \left( \pm \pi i \vartheta_{j}\right), \quad\left(\mathcal{M}_{3} \mathcal{M}_{2} \mathcal{M}_{1}\right)^{-1}=\mathcal{M}_{\infty}, \quad \text { eigen }\left(\mathcal{M}_{\infty}\right)=\exp \left( \pm \pi i \vartheta_{\infty}\right), \\
\mathcal{M}_{k} \neq \pm \mathbf{1} \quad \forall k=1,2,3, \infty,
\end{gathered}
$$

with some numbers $\vartheta_{1}, \vartheta_{2}, \vartheta_{3}, \vartheta_{\infty}$, and $\mathcal{R}_{1}, \mathcal{R}_{2}, \mathcal{R}_{3}$ satisfying (2.14). If there exists a branch of a solution of the PVI equation with parameters (2.28) such that the Fuchsian system of the form (2.1) given by (2.24) has the prescribed monodromy data $\left\{\left(\mathcal{M}_{1}, \mathcal{M}_{2}, \mathcal{M}_{3}\right) / \sim, \mathcal{R}_{1}, \mathcal{R}_{2}, \mathcal{R}_{3}\right\}$ then this branch is unique modulo symmetries.

\section{Analytic continuation and rational solutions of the Painlevé VI equation.}

In Theorem 2.10, we parameterised branches of generic solutions of PVI by triples of monodromy matrices. Following $[\mathrm{DM}]$, now we show how these parameters change with a change of the branch in the process of analytic continuation of the solutions along a path in $\overline{\mathbb{C}} \backslash\{0,1, \infty\}$. Recall that, as it follows from Theorem 2.5, any solution of the Schlesinger equations can be continued analytically from a point $\left(u_{1}^{0}, u_{2}^{0}, u_{3}^{0}\right)$ to another point $\left(u_{1}^{1}, u_{2}^{1}, u_{3}^{1}\right)$ along a path

$$
\left(u_{1}(t), u_{2}(t), u_{3}(t)\right) \in \mathbb{C}^{3} \backslash\{\text { diags }\}, \quad 0 \leq t \leq 1,
$$

where $\{$ diags $\}=\left\{u_{1}, u_{2}, u_{3} \mid u_{i}=u_{j}\right.$, for some $\left.i \neq j\right\}$ and

$$
u_{i}(0)=u_{i}^{0}, \quad \text { and } \quad u_{i}(1)=u_{i}^{1},
$$

provided that the end-points are not the poles of the solution. The result of the analytic continuation depends only on the homotopy class of the path in $\mathbb{C}^{3} \backslash\{$ diags $\}$. Particularly, to find all the branches of a solution near a given point $u^{0}=\left(u_{1}^{0}, u_{2}^{0}, u_{3}^{0}\right)$ one has to compute the results of the analytic continuation along any homotopy class of closed loops in $\mathbb{C}^{3} \backslash\{$ diags $\}$ with the beginning and the end at the point $u^{0}=\left(u_{1}^{0}, u_{2}^{0}, u_{3}^{0}\right)$. Let

$$
\beta \in \pi_{1}\left(\mathbb{C}^{3} \backslash\{\text { diags }\} ; u^{0}\right)
$$

be an arbitrary loop. Any solution of the Schlesinger equations near the point $u^{0}=\left(u_{1}^{0}, u_{2}^{0}, u_{3}^{0}\right)$, is uniquely determined up to (2.23) by the monodromy matrices $\mathcal{M}_{1}, \mathcal{M}_{2}$ and $\mathcal{M}_{3}$, computed with respect to the basis of loops $\gamma_{1}, \gamma_{2}, \gamma_{3}$. Continuing analytically this solution along the loop $\beta$, we arrive at another branch of the same solution near $u^{0}$. This new branch is specified, according to Theorem 2.10, by some new monodromy matrices $\mathcal{M}_{1}^{\beta}, \mathcal{M}_{2}^{\beta}$ and $\mathcal{M}_{3}^{\beta}$, computed in the same basis $\gamma_{1}, \gamma_{2}, \gamma_{3}$. We want to compute these new matrices for any loop $\beta \in \pi_{1}\left(\mathbb{C}^{3} \backslash\{\right.$ diags $\left.\} ; u^{0}\right)$. The fundamental group $\pi_{1}\left(\mathbb{C}^{3} \backslash\{\right.$ diags $\left.\} ; u^{0}\right)$ is isomorphic to the pure (or unpermuted) braid group, $P_{3}$ with three strings (see $[\mathrm{Bir}]$ ). 
Lemma 3.1. For the generators $\beta_{1}, \beta_{2}, \beta_{3}$ of the pure braid group $P_{3}, \mathcal{M}_{i}^{\beta}$ have the following form:

$$
\begin{gathered}
\mathcal{M}_{1}^{\beta_{1}}=\mathcal{M}_{1} \mathcal{M}_{2} \mathcal{M}_{1} \mathcal{M}_{2}^{-1} \mathcal{M}_{1}^{-1}, \quad \mathcal{M}_{2}^{\beta_{1}}=\mathcal{M}_{1} \mathcal{M}_{2} \mathcal{M}_{1}^{-1}, \quad \mathcal{M}_{3}^{\beta_{1}}=\mathcal{M}_{3}, \\
\mathcal{M}_{1}^{\beta_{2}}=\mathcal{M}_{1} \mathcal{M}_{3} \mathcal{M}_{1} \mathcal{M}_{3}^{-1} \mathcal{M}_{1}^{-1}, \quad \mathcal{M}_{3}^{\beta_{2}}=\mathcal{M}_{1} \mathcal{M}_{3} \mathcal{M}_{1}^{-1} \\
\mathcal{M}_{2}^{\beta_{2}}=\mathcal{M}_{1} \mathcal{M}_{3} \mathcal{M}_{1}^{-1} \mathcal{M}_{3}^{-1} \mathcal{M}_{2} \mathcal{M}_{3} \mathcal{M}_{1} \mathcal{M}_{3}^{-1} \mathcal{M}_{1}^{-1} \\
\mathcal{M}_{1}^{\beta_{3}}=\mathcal{M}_{1} \quad \mathcal{M}_{2}^{\beta_{3}}=\mathcal{M}_{2} \mathcal{M}_{3} \mathcal{M}_{2} \mathcal{M}_{3}^{-1} \mathcal{M}_{2}^{-1}, \quad \mathcal{M}_{3}^{\beta_{3}}=\mathcal{M}_{2} \mathcal{M}_{3} \mathcal{M}_{2}^{-1}
\end{gathered}
$$

Proof. This lemma is proved in $[D M]$.

Lemma 3.2. If a solution of the Painlevé VI equation such that none of the corresponding monodromy matrices $\mathcal{M}_{1}, \mathcal{M}_{2}, \mathcal{M}_{3}$ are multiples of the identity is rational then $\mathcal{M}_{1}, \mathcal{M}_{2}, \mathcal{M}_{3}$ are fixed points under the above action (2.29), (2.30), (2.31) on the space of triples

$$
\left\{\left(\mathcal{M}_{1}, \mathcal{M}_{2}, \mathcal{M}_{3}\right) / \sim, \mathcal{M}_{3} \mathcal{M}_{2} \mathcal{M}_{1}=\mathcal{M}_{\infty}^{-1}\right\}
$$

the equivalence relation $\sim$ being defined in (2.12).

Proof. The action (2.29), (2.30), (2.31) of the pure braid group on the triples of monodromy matrices not only describes the structure of the analytic continuation of the solutions of the Schlesinger equations (2.22), but also of the reduced ones (2.25) and thus of the PVI equation. A necessary condition for a solution to be rational is that it has only one branch. Since the action (2.29), (2.30), (2.31) of the pure braid group on the triples of monodromy matrices commutes with the conjugation (2.12), the triple of monodromy matrices $\mathcal{M}_{1}, \mathcal{M}_{2}, \mathcal{M}_{3}$ corresponding to a rational solution is unique up to (2.12).

Lemma 3.3. If a solution of PVI such that none of the corresponding monodromy matrices $\mathcal{M}_{1}, \mathcal{M}_{2}, \mathcal{M}_{3}$ are multiples of the identity is rational then the monodromy matrices all commute

$$
\left[\mathcal{M}_{i}, \mathcal{M}_{j}\right]=0, \quad \forall i, j=1,2,3 .
$$

Proof. By Lemma 3.2, we have to impose

$$
\begin{aligned}
& L_{\infty 1}{ }_{1}^{-1} \mathcal{M}_{1} L_{\infty 1}=\mathcal{M}_{1}^{\beta_{1}}=\mathcal{M}_{1} \mathcal{M}_{2} \mathcal{M}_{1} \mathcal{M}_{2}^{-1} \mathcal{M}_{1}^{-1} \\
& L_{\infty 1}{ }_{1}^{-1} \mathcal{M}_{2} L_{\infty 1}=\mathcal{M}_{2}^{\beta_{1}}=\mathcal{M}_{1} \mathcal{M}_{2} \mathcal{M}_{1}^{-1} \\
& L_{\infty 1}{ }^{-1} \mathcal{M}_{3} L_{\infty 1}=\mathcal{M}_{3}^{\beta_{1}}=\mathcal{M}_{3} \\
& L_{\infty 2}{ }^{-1} \mathcal{M}_{1} L_{\infty 2}=\mathcal{M}_{1}^{\beta_{2}}=\mathcal{M}_{1} \mathcal{M}_{3} \mathcal{M}_{1} \mathcal{M}_{3}^{-1} \mathcal{M}_{1}^{-1} \\
& L_{\infty 2}{ }^{-1} \mathcal{M}_{2} L_{\infty 2}=\mathcal{M}_{2}^{\beta_{2}}=\mathcal{M}_{1} \mathcal{M}_{3} \mathcal{M}_{1}^{-1} \mathcal{M}_{3}^{-1} \mathcal{M}_{2} \mathcal{M}_{3} \mathcal{M}_{1} \mathcal{M}_{3}^{-1} \mathcal{M}_{1}^{-1} \\
& L_{\infty 2}{ }^{-1} \mathcal{M}_{3} L_{\infty 2}=\mathcal{M}_{3}^{\beta_{2}}=\mathcal{M}_{1} \mathcal{M}_{3} \mathcal{M}_{1}^{-1} \\
& L_{\infty 3}{ }^{-1} \mathcal{M}_{1} L_{\infty 3}=\mathcal{M}_{1}^{\beta_{3}}=\mathcal{M}_{1} \\
& L_{\infty}{ }^{-1} \mathcal{M}_{2} L_{\infty 3}=\mathcal{M}_{2}^{\beta_{3}}=\mathcal{M}_{2} \mathcal{M}_{3} \mathcal{M}_{2} \mathcal{M}_{3}^{-1} \mathcal{M}_{2}^{-1} \\
& L_{\infty}{ }^{-1} \mathcal{M}_{3} L_{\infty 3}=\mathcal{M}_{3}^{\beta_{3}}=\mathcal{M}_{2} \mathcal{M}_{3} \mathcal{M}_{2}^{-1}
\end{aligned}
$$

for some suitable matrices $L_{\infty 1}, L_{\infty 2}, L_{\infty 3}$ that are diagonal for $\vartheta_{\infty} \notin \mathbb{Z}$, are in Jordan form for $\vartheta_{\infty} \in \mathbb{Z}$ and $\mathcal{R}_{\infty} \neq 0$. Then we have to distinguish two cases: i) $\mathcal{M}_{\infty} \neq \pm \mathbf{1}$ is diagonal, ii) $\mathcal{M}_{\infty} \neq \pm \mathbf{1}$ is in Jordan form.

i) In this case, the matrices $L_{\infty 1}, L_{\infty 2}, L_{\infty 3}$ are diagonal. If none of the matrices $\mathcal{M}_{1,2,3}$ is diagonal in the basis of $\mathcal{M}_{\infty}$ diagonal, then the above matrices $L_{\infty 1}, L_{\infty 3}$ must be chosen to be multiples of the identity. Thus, from

$$
\begin{aligned}
& \mathcal{M}_{2}=\mathcal{M}_{2}^{\beta_{1}}=\mathcal{M}_{1} \mathcal{M}_{2} \mathcal{M}_{1}^{-1}, \\
& \mathcal{M}_{3}=\mathcal{M}_{3}^{\beta_{3}}=\mathcal{M}_{2} \mathcal{M}_{3} \mathcal{M}_{2}^{-1},
\end{aligned}
$$


it follows immediately that

$$
\left[\mathcal{M}_{2}, \mathcal{M}_{1}\right]=0, \quad \text { and } \quad\left[\mathcal{M}_{2}, \mathcal{M}_{3}\right]=0
$$

thus

$$
L_{\infty 2}^{-1} \mathcal{M}_{2} L_{\infty 2}=\mathcal{M}_{2}^{\beta_{2}}=\mathcal{M}_{2}
$$

i.e. $L_{\infty 2}$ is the identity matrix as well and thus we obtain (2.32). If one of the monodromy matrices $\mathcal{M}_{1,2,3}$ is diagonal in the basis of $\mathcal{M}_{\infty}$ diagonal, for example $\mathcal{M}_{1}$, then either $L_{\infty 1}$ is the identity matrix or $\mathcal{M}_{3}$ is diagonal. If $L_{\infty 1}$ is the identity matrix, from (2.33) we have that $\mathcal{M}_{2}$ is diagonal as well, and being $\mathcal{M}_{\infty}$ diagonal as well, $\mathcal{M}_{3}$ must be diagonal and we find again (2.32). If $\mathcal{M}_{3}$ and $\mathcal{M}_{1}$ are both diagonal, since $\mathcal{M}_{\infty}$ is diagonal, $\mathcal{M}_{2}$ must be diagonal too and we find again (2.32).

ii) Suppose that $\mathcal{M}_{\infty}$ is in Jordan form. Then the matrices $L_{\infty 1}, L_{\infty 2}, L_{\infty 3}$ have the form $\left(\begin{array}{cc}1 & a_{i} \\ 0 & 1\end{array}\right)$ for some constants $a_{1}, a_{2}, a_{3}$. If none of the matrices $\mathcal{M}_{1}, \mathcal{M}_{2}, \mathcal{M}_{3}$ is in Jordan form, all matrices $L_{\infty 1}, L_{\infty 2}, L_{\infty 3}$ are the identity matrix and we obtain (2.32) as above. Suppose that one of the monodromy matrices, for example $\mathcal{M}_{1}$ is in Jordan form. Then, either $L_{\infty 1}$ is the identity and $\left[\mathcal{M}_{2}, \mathcal{M}_{1}\right]=0$ or $\mathcal{M}_{\ni}$ is in Jordan form. Reasoning as above we obtain that all matrices $\mathcal{M}_{1}, \mathcal{M}_{2}, \mathcal{M}_{3}$ are in Jordan form and thus commute. $\triangle$

\section{Reducible monodromy groups.}

Theorem 4.1. All solutions of PVI corresponding to reducible monodromy groups are equivalent via birational canonical transformations and symmetries to the following one-parameter family of solutions, realized for $\vartheta_{\infty}=-\left(\vartheta_{1}+\vartheta_{2}+\vartheta_{3}\right)$

$$
y=\frac{\left(1+\vartheta_{1}+\vartheta_{2}-x-\vartheta_{2} x\right) u(x)-x(x-1) u_{x}(x)}{\left(1+\vartheta_{1}+\vartheta_{2}+\vartheta_{3}\right) u(x)}
$$

where $u(x)=u_{1}(x)+\nu u_{2}(x), u_{1}(x), u_{2}(x)$ being two linear independent solutions of the following hypergeometric equation

$$
x(1-x) u_{x x}(x)+\left[2+\vartheta_{1}+\vartheta_{2}-\left(4+\vartheta_{1}+2 \vartheta_{2}+\vartheta_{3}\right) x\right] u_{x}(x)-\left(2+\vartheta_{1}+\vartheta_{2}+\vartheta_{3}\right)\left(\vartheta_{2}+1\right) u(x)=0 .
$$

Proof. For reducible monodromy groups there exists a basis in which all monodromy matrices are upper triangular. We can always perform a change of basis in order that $\mathcal{M}_{\infty}$ has the form (2.8) and all monodromy matrices have the form:

$$
\mathcal{M}_{k}=\left(\begin{array}{cc}
\exp \left(\pi i \vartheta_{k}\right) & \star \\
0 & \exp \left(-\pi i \vartheta_{k}\right)
\end{array}\right) .
$$

It then follows, by the relation (2.11), that $\vartheta_{\infty}+\varepsilon_{k} \sum_{k} \vartheta_{k}=2 N, \varepsilon_{k}= \pm 1, N \in \mathbb{Z}$. By means of the birational canonical transformations and symmetries of the PVI equation, we can always assume that $\varepsilon_{k}=+1$ and $N=0$. Perform the following gauge transformation on the Fuchsian system

$$
\Phi \rightarrow \tilde{\Phi}=\Pi_{k=1}^{3}\left(\lambda-u_{k}\right)^{\frac{-\vartheta_{k}}{2}} \Phi, \quad \mathcal{A}_{k} \rightarrow \tilde{\mathcal{A}}_{k}=\mathcal{A}_{k}-\frac{\vartheta_{k}}{2} \mathbf{1} .
$$

The new residue at infinity is

$$
\tilde{\mathcal{A}}_{\infty}=\left(\begin{array}{cc}
0 & 0 \\
0 & \frac{-\vartheta_{\infty}+\sum \vartheta_{k}}{2}
\end{array}\right)
$$

and the new monodromy matrices are

$$
\tilde{\mathcal{M}}_{k}=\exp \left(-\pi i \vartheta_{k}\right) \mathcal{M}_{k}=\left(\begin{array}{cc}
1 & \star \\
0 & \exp \left(-2 \pi i \vartheta_{k}\right)
\end{array}\right), \quad k=1,2,3,
$$


and

$$
\tilde{\mathcal{M}}_{\infty}=\left(\begin{array}{cc}
1 & 0 \\
0 & \exp \left[\pi i\left(-\vartheta_{\infty}+\sum_{k} \vartheta_{k}\right)\right]
\end{array}\right) \exp \left(\mathcal{R}_{\infty}\right)
$$

Since all monodromy matrices have the first column given by $\left(\begin{array}{l}1 \\ 0\end{array}\right)$, the new Fuchsian system admits a nonzero single valued vector solution $\tilde{Y}$. This solution is analytic at $u_{1}, u_{2}$ and $u_{3}$ because all residue matrices $\tilde{\mathcal{A}}_{k}, k=1,2,3$ have a zero eigenvalue. At infinity $\tilde{Y}$ is necessarily constant. Thus, near each $u_{j}$, one has

$$
\frac{\tilde{\mathcal{A}}_{j}}{\lambda-u_{j}} \tilde{Y}+\mathcal{O}(1)=0
$$

that implies that $\tilde{Y}$ has the form $\left(\begin{array}{l}a \\ 0\end{array}\right)$, for some $a \neq 0$ and all residue matrices $\mathcal{A}_{k}$ are upper triangular. Correspondingly $p=\sum_{k=1}^{3} \frac{\vartheta_{k}}{\left(q-u_{k}\right)}$ and the solution $y(x)$ of PVI is given by (4.1).

Remark 4.2. Observe that the appearance of the hypergeometric equation in Theorem 4.1 is quite natural. In fact, when all the residue matrices $\mathcal{A}_{k}$ are upper-triangular, the Schlesinger equations, and thus the Painlevé VI equation, linearise.

Lemma 4.3. The one-parameter family of classical solutions (4.1) contains at least one rational solution if and only if one of the values $\vartheta_{1}, \vartheta_{2}, \vartheta_{3}, \vartheta_{\infty}$ is an integer.

Proof. The one-parameter family of classical solutions (4.1) contains at least one algebraic solution if and only if the corresponding Riccati equation

$$
y_{x}(x)=\frac{1+\vartheta_{1}+\vartheta_{2}+\vartheta_{3}}{x(x-1)} y^{2}-\frac{1+\vartheta_{1}+\vartheta_{2}+\vartheta_{1} x+\vartheta_{3} x}{x(x-1)} y+\frac{\vartheta_{1}}{x-1}
$$

has at least one algebraic solution, i.e., if and only the corresponding hypergeometric equation (4.2) is integrable in the sense of differential Galois theory (see [Mor]). This happens if and only if and only if the parameters $\lambda, \mu, \nu$ of the hypergeometric equation belong to the Schwartz-Kimura table (see [Mor]). In particular rational solutions are occur only when at least one of the numbers $\mu-\lambda+\nu, \mu-\lambda-\nu, \mu+\lambda+\nu$, $\mu+\lambda-\nu$ is an odd integer. In our case $\lambda=-1-\vartheta_{1}-\vartheta_{2}, \mu=1+\vartheta_{1}+\vartheta_{3}, \nu=-1-\vartheta_{3}-\vartheta_{2}$ so the one-parameter family of classical solutions (4.1) contains at least one rational solution if and only if one of the values $\vartheta_{1}, \vartheta_{2}, \vartheta_{3}, \vartheta_{\infty}$ is an integer.

To conclude the proof of the main theorem, we use the following lemma

Lemma 4.4. All solutions of PVI such that one or more monodromy matrices $\mathcal{M}_{1}, \mathcal{M}_{2}, \mathcal{M}_{3}, \mathcal{M}_{\infty}$ is a multiple of the identity are either degenerate or are equivalent via birational canonical transformations to the one-parameter family (4.1) of Theorem 4.1.

Proof. We omit the proof of this Lemma that can be found for example in [Ma1].

The classification is concluded observing that if one or more of the monodromy matrices is a multiple of the identity, then the corresponding solution is equivalent via birational canonical transformations to a solution with reducible monodromy groups. If none of the monodromy matrices is a multiple of the identity, then in order to have a rational solution the monodromy group must be Abelian. All Abelian $2 \times 2$ monodromy groups are reducible. Thus all rational non-degenerate solutions of the PVI equation belong to the one parameter family of classical solutions given in Theorem 4.1, up to birational canonical transformations. Then we can use Lemma 4.3 to classify them.

\section{BIBLIOGRAPHY}

[Ai] H. Airault, Rational solutions of Painlevé equations, Stud. Appl. Math. 61, (1979) 31-53.

[AMM] D.A. Albrecht, E.L. Mansfield, A.E. Milne, Algoritms for special integrals of ordinary differential equations, J. Phys. A: Math. Gen. 29 (1996) 973-991. 
[Bir] J.S. Birman, Braids, Links, and Mapping Class Groups, Ann. Math. Stud. Princeton University (1975).

[Dub] B. Dubrovin, Painlevé Transcendents in Two-Dimensional Topological Field Theory, The Painlevé Property One Century Later, Robert Conte editor, CRM Series in Mathematical Physics, (1999).

[DM] B. Dubrovin, M.Mazzocco, Monodromy of certain Painlevé VI transcendents and Reflection Groups, Invent. Math., 141 (2000) 55-147.

[FIN] H. Flashka and A.C. Newell, Monodromy and Spectrum Preserving Deformations, Comm. Math. Phys. 76 (1980) 67-116.

[Fuchs] R. Fuchs, Über Lineare Homogene Differentialgleichungen Zweiter Ordnung mit im drei im Endrichen Gelegene Wesentlich Singulären Stellen, Math. Ann. 63 (1907) 301-321.

[Gam] B. Gambier, Sur les Equations Differentielles du Second Ordre et du Primier Degrè dont l'Integrale est a Points Critiques Fixes, Acta Math. 33, (1910) 1-55.

[Gar] R. Garnier, Sur des Equations Differentielles du Troisieme Ordre dont l'Integrale Generale est Uniforme et sur una Classe d'Equations nouvelles d'Ordre Superieur dont l'Integrale Generale a ses Points Critiques Fixes, Ann. Sci. Ecole Norm. Sup. 3 No.29 (1912) 1-126.

[Gr] V.I. Gromak, Single-parameter families of solutions of Painlevé equations, Diff. Eqns. 14 (1978), $1510-1513$.

[GL] V.I. Gromak, N.A. Lukashevich, Special classes of solutions of Painlevé equations, Diff. Eqns. 18 (1982) $317-326$.

[Hit] N. Hitchin, Hypercomplex Manifolds and the Space of Framings, The Geometric Universe, Oxford Univ. Press, Oxford, 1998, 9-30.

[Ince] E.L. Ince, Ordinary Differential Equations, Dover Publications, New York (1956).

[ItN] A.R. Its and V.Yu. Novokshenov, The Isomonodromic Deformation Method in the Theory of Painlevé Equations, Springer Lect. Notes Math. 1191 (1986).

[JMU] M. Jimbo, T. Miwa and K. Ueno, Monodromy Preserving Deformation of the Linear Ordinary Differential Equations with Rational Coefficients I, II, III, Physica D, 2 (1981), no. 2, 306-352, 2 (1981), no. 3, 407-448, 4 (1981/82), no. 1, 26-46.

[Luk] N.A. Lukashevich, Elementary solutions of certain Painlevé equations, Differ. Uravn. 1 (1965) 731-735.

[Mal] B. Malgrange, Sur les Deformations Isomonodromiques I, Singularités Régulières, Seminaire de l'Ecole Normale Superieure 1979-1982, Progress in Mathematics 37, Birkhäuser, Boston (1983) 401-426.

[Ma] M. Mazzocco, Picard and Chazy Solutions to PVI Equation, Math. Ann., 321 (2001), 157-195.

[Ma1] M. Mazzocco, The geometry of the classical solutions of the Garnier systems, I.M.R.N., 12 (2002) 613-646.

[Miwa] T. Miwa, Painlevé Property of Monodromy Preserving Deformation Equations and the Analyticity of $\tau$-function, Publ. RIMS, Kyoto Univ. 17 (1981) 703-721.

[Mor] J.J. Morales, Differential Galois theory and non-integrability of Hamiltonian systems Progress in Mathematics, 179, (1999).

[Ok] K. Okamoto, Studies on the Painlevé Equations I, Sixth Painlevé Equation, Ann. Mat. Pura Appl. 146 (1987) 337-381.

[Pain] P. Painlevé, Sur les Equations Differentielles du Second Ordre et d'Ordre Superieur, dont l'Interable Generale est Uniforme, Acta Math. 25 (1902) 1-86.

[Sch] L. Schlesinger, Über eine Klasse von Differentsial System Beliebliger Ordnung mit Festen Kritischer Punkten, J. fur Math. 141 (1912), 96-145. 
[Schw] H.A. Schwartz, Über Diejenigen Fälle in Welchen die Gaussische Hypergeometrische Reihe einer Algebraische Funktion iheres vierten Elementes Darstellit, Crelle J. 75 (1873) 292-335.

[Sib] Y. Sibuya, Linear Differential Equations in the Complex Domain: Problems of Analytic Continuation, AMS TMM 82 (1990).

[Um] H. Umemura, Special polynomials associated with the Painlevé equations I, to appear in Painlevé transcendents, CRM Montreal, Canada (1996).

[Um1] H. Umemura, Irreducebility of the First Differential Equation of Painlevé, Nagoya Math. J., 117 (1990) $231-252$.

[Um2] H. Umemura, Second proof of the irreducebility of the First Differential Equation of Painlevé, Nagoya Math. J., 117 (1990) 125-171.

[Wat] H. Watanabe, Birational Canonical Transformations and Classical Solutions of the Sixth Painlevé Equation, Ann. Scuola Norm. Sup. Pisa Cl. Sci. 27 (1998), no. 3-4, 379-425 (1999). 